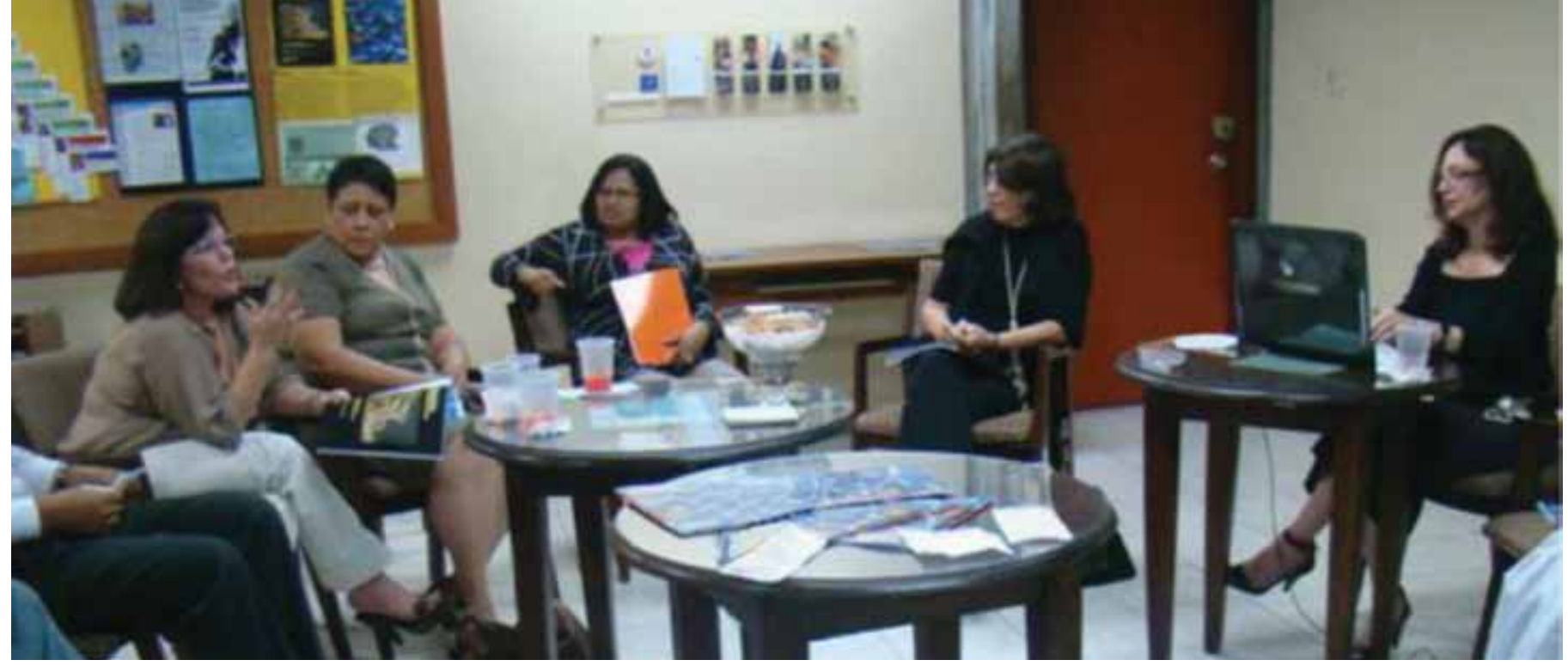

\title{
LA IX PEÑA PEDAGÓGICA
}

Estos encuentros, de carácter informal entre el profesorado de la PUCMM, se organizan dos veces al año para compartir ideas y opiniones sobre los artículos del último ejemplar del Cuaderno de Pedagogía Universitaria. El tema anterior fue "Equipos docentes: prácticas y reflexiones" y las reuniones se llevaron a cabo el 3 y el 17 de noviembre de 2010, en Santiago y en Santo Domingo. A continuación reseñamos las ideas principales.

\section{En Santiago}

Como es costumbre, en estos encuentros participan los articulistas que escribieron en el ejemplar anterior. No obstante, en esta ocasión, como estos residen en el extranjero o forman parte de otras universidades dominicanas con sede en Santo Domingo, solo se contó con la participación de Nora Ramírez, quien habló sobre el artículo publicado desde el Centro de Desarrollo Profesoral (CDP) de la PUCMM. También estuvo presente Ana Margarita Haché, la Directora anterior del CDP, que se refirió al seminario inter-universitario celebrado en diciembre de 2009 con el tema de "Los equipos docentes: una modalidad de buenas prácticas", evento a partir del cual surgió la motivación de dedicar el Cuaderno No. 13 a la publicación de las ponencias presentadas en el mismo.

El diálogo lo inició un profesor de Estomatología que asiste siempre a la Peña diciendo que, en cada lectura de un nuevo número del Cuaderno, reordena la secuencia de los artículo. Sin embargo, esta vez consideró que la organización era la ideal. Señaló que el artículo de Miguel Ángel Zabalza introducía al ámbito conceptual desarrollado en el Cuaderno. Cada artículo se complementaba, pues el tema no tratado en uno se encontraba desarrollado en otro. Añadió que la lectura del Cuaderno siempre lo inquieta, pues lo hace reflexionar sobre tantas cosas que quedan por hacer en la Universidad. Esta sensación, sin duda, está también para él presente en las Peñas, espacio donde se debate el compromiso de todos los actores de la universidad por la mejora de la calidad educativa y cuyo valor reside, según este profesor, en el encuentro cara a cara. Para ilustrar su idea, contrastó su experiencia en los debates orales que tenían lugar en el departamento con el intercambio actual de ideas que se lleva a cabo ahora mediado por la tecnología. Destacó que a pesar de esta ser muy efectiva y de ahorrar tiempo, la riqueza del debate oral consiste en unir e identificar al profesorado con la Institución. Otro profesor presente, de Ingeniería Industrial, corroboró la importancia de la identificación con la Universidad dando como ejemplo la relación que se había desarrollado en el grupo de profesores egresados de la Especialidad (EPU) y la Maestría (MPU) en Pedagogía de la PUCMM a partir de la dinámica de trabajo que se propone en dichos estudios.

Las ideas expuestas por los demás fueron apoyadas por un profesor de Arquitectura que asistió por primera vez a la Peña. Para él, el artículo de Zabalza es un resumen del programa de estudios del Diplomado en Pedagogía Universitaria de esta Universidad del cual es estudiante. Si cada profesor se convence de tomar acción, sin esperar que el cambio venga desde arriba, se pueden echar a andar un sinnúmero de cosas. Se aludió a la temática del Cuaderno, el apoyo al trabajo en equipo, y se recalcó que si bien es cierto que emprender proyectos en conjunto es difícil, los beneficios a la larga son mayores que el trabajo solitario. Indicaron que es esto lo que se quiere también llevar a las aulas, entre los estudiantes.

Con respecto a este último tema, se dijo que, de hecho, existen iniciativas valiosas de trabajo en equipos docentes. Se señaló, además de la experiencia de Estomatología antes mencionada, el trabajo en coordinación que se hace en algunas asignaturas de Arquitectura con egresados de la EPU y la MPU. Sin duda, sería muy constructivo para la Universidad dar a conocer los datos sobre la incidencia de la formación de equipos entre los egresados de los programas de formación docente de la PUCMM.

Una profesora de Humanidades confirmó que, aunque el profesorado de este Departamento no ha cursado los programas de formación pedagógica de la Universidad, se ha visto en los últimos años una fuerte tendencia al trabajo en equipo. Estos profesores no sólo se ponen de acuerdo con el contenido de los programas sino también en el diseño de estrategias, lo que los lleva a crecer juntos comparando ideas y construyendo otras nuevas. No siempre estas iniciativas son del conocimiento de todos en la Universidad, pero aunque no se difundan no quiere decir que no existen. Esta idea se fundamentó con el artículo escrito por Laura Lodeiro, doctoranda de la Universidad de Santiago de Compostela. En su artículo ella hablaba sobre diferentes 
modalidades de trabajo con otros, es decir, iniciativas de equipo que van desde la unión espontánea de los profesores mismos, hasta la concepción de universidades que nacen con un diseño cooperativo en el currículo y en la gestión.

También el nuevo Director de Educación, presente en la Peña, confirmó la existencia del trabajo en colaboración entre los profesores de Humanidades. Relató que los equipos se reúnen para discutir los exámenes que van a llevar a los estudiantes. Se ha pasado de la modalidad de que el Departamento exigía un mismo examen para todos los grupos hasta una forma más flexible donde cada profesor crea su propio examen, lo comparte y los demás se enriquecen de las ideas que les interese introducir o cambiar en el suyo. Este Director también contó la experiencia de grupos de discusión entre los estudiantes de pregrado, con temas amplios de interés general. Es muy satisfactorio para él ver que los estudiantes no se quieren ir de este tipo de encuentros y motivó para que los mismos se realicen entre profesores, para compartir temáticas especificas desde sus perspectivas disciplinares.

El diálogo se enriqueció con la participación de la nueva Directora de Psicología. Expuso la idea de que todas esas experiencias tan ricas pueden ser identificadas por los Directores Departamentales para sugerir articulistas al Cuaderno de Pedagogía. Opinó que escribir en el Cuaderno debería ser un honor, un reto. También sugirió que otra buena iniciativa es la de promover pequeñas Peñas en los Departamentos para discutir sobre el Cuaderno y generar temas de escritura. El reto es el de continuar apoyando las alianzas estrechas entre el Centro de Desarrollo Profesoral y los Departamentos. Las prácticas nuevas toman tiempo instalarlas e institucionalizarlas, pero la perseverancia es la mejor herramienta.

Durante la Peña se aportaron sugerencias para el Cuaderno. Los participantes recomendaron la inclusión de los nombres de las personas presentes en este encuentro al final de la escritura de esta relatoría, así como la redefinición del sistema de distribución del Cuaderno entre el profesorado de la Universidad. Muchos profesores leen el Cuaderno, pero es cierto que muchos no lo hacen, un cambio en la forma en la que se distribuye podría aumentar la incidencia de lectura.

La Peña terminó con muchas propuestas de actividades que los profesores pueden hacer para mejorar la calidad educativa de la Institución. A partir de Diciembre 2010 se va a reabrir en la Universidad la Carrera Docente. Es una buena oportunidad para rediseñar el sistema contractual del profesorado, de manera que su trabajo pueda diversificarse en líneas de acción que hagan crecer la Institución por nuevas vías. Según las ideas compartidas, en esta ocasión el ambiente de la Peña fue propositivo, con la esperanza y la motivación de seguir construyendo una mejor PUCMM.

Asistentes a la Peña: Ariosto Díaz, Estomatología; Mauricio Estrella, Arquitectura; Yngris Balbuena, Lingüística; Annery Abreu, Humanidades; Johnny Mezón, Ingeniería Industrial; Claudette García, Psicología; Juan Zapata, Educación; Ana Margarita Haché, asesora del Cuaderno de Pedagogía Universitaria; Nora Ramírez, Sandra Hernández, Iván Carrasco y Rosario Corominas, Centro de Desarrollo Desarrollo Profesoral.

\section{En Santo Domingo}

La Peña inició con la contextualización del Cuaderno de Pedagogía No.13, recordando el Seminario Interuniversitario "Los equipos docentes: una modalidad de buenas prácticas" realizado en el campus de Santiago en diciembre de 2009. La invitación inicial a los participantes fue la de reaccionar ante la lectura amplia del cuaderno, por ejemplo, qué señales nos da, qué reflexiones genera.

Uno de los asistentes indicó la trascendencia del editorial como aspecto a resaltar del Cuaderno. Indicaba que desde la Especialidad en Pedagogía Universitaria, ofrecida por la Universidad a su profesorado, se ha invitado a reflexionar sobre la práctica docente. $Y$, de hecho, uno de los temas a desarrollar ha sido el trabajo colaborativo, lo cual se hace explícito en el editorial. Este profesor enfatizó cómo hoy día la sociedad no promueve el trabajo autónomo y, en este sentido, el editorial resalta el papel de toda universidad en hacer énfasis sobre los equipos de trabajo, por que es una manera de que los docentes potenciemos, a su vez, esa dimensión en nuestro estudiantado. Al leer el editorial entre todos en la Peña, se generaron cuestionantes sobre si las actividades académicas llevan a fomentar el trabajo de equipo en el aula.

Uno de los asistentes señaló que en el artículo de Zabalza, este autor plantea que la enseñanza debe ir desde lo teórico a lo práctico, con una evaluación de la ganancia que van a recibir los estudiantes con el enfoque por competencias. Algunas reacciones sobre el artículo en los demás asistentes apuntaban a que Zabalza vive en España un momento de transición hacia un currículo universitario por competencias, con las dificultades inherentes a toda innovación. En España buscan implementar un nuevo currículo que conlleva unas prácticas colectivas y, por lo tanto, sale a relucir el contraste entre lo individualizado y lo colaborativo.

La reflexión grupal continuó en torno a las modalidades del trabajo en equipo planteadas por Zabalza. Hay una modalidad de trabajo en equipo que es impuesta por la institución, pero no es la más adecuada. El ser humano, frente a lo impuesto tiene como primera opción el rebelarse, cuestiona y se va a favor de la autonomía. La otra modalidad que se plantea es lo interdisciplinario. Todos somos un conjunto, para desde ahí poder trabajar y desarrollar entre todos un currículo basado en competencias. Es una pérdida de tiempo cuando no estamos todas las disciplinas enfocadas hacia allá.

Un profesor indicó que no le quedaba claro cuándo la universidad decide fragmentar el pensamiento y el conocimiento en "pedacitos". Resaltó cómo la realidad de la Educación Superior hoy es la de un grupo de especialistas que se van aislando y aislando, con un enfoque donde "lo importante es mi materia y las otras no". Sugirió que existan niveles de mediación que revisen esa dinámica y aseguren una labor integradora. La tendencia es que cada uno desde nuestra área aporte para articular; uno solo no puede, son todas las disciplinas las que deben involucrarse, señaló.

Una profesora resaltó que la primera vez que se habló de la pedagogía del docente universitario en la Institución, el Lic. Francisco Polanco (antiguo Vicerrector Académico) planteaba "el ánimo no es que todos sean pedagogos inicialmente, sino que primero nos 
juntemos, seamos uno". La pedagogía es de un grupo, hagamos un programa que permita ese rol, asumir ese rol grupal. Otra reflexión de la profesora fue en dirección al Seminario en Estudios Generales realizado semanas antes en el Campus de Santiago. Indicó que la mayoría de las personas participantes del seminario imparten clases en el Ciclo Básico. Los especialistas no fueron y eso dificulta la posibilidad de interactuar, pues se pierde la comunicación cuando cada uno baja la cabeza y se queda en lo suyo. Finalmente, esta profesora apuntó que contra la convergencia europea y el Acuerdo de Bolonia hay una rebelión pues les están haciendo una "caja donde deben entrar" y hay mucha rebeldía. Lo que han buscado como respuesta es otro enfoque de competencias, desde un enfoque más holístico.

Se compartió la idea de lo difícil que es ver al profesor trabajando de manera aislada, independiente, pues el profesor es parte de un todo que es la universidad, la cual le traza un programa, unos lineamientos. En el grupo surgió entonces la interrogante del rol de las autoridades universitarias para incentivar, promover el trabajo en equipo desde la misma estructura de la Universidad, por medio de una labor desde los Departamentos y cómo esa visión debe llegar hasta los docentes y su accionar.

Vivimos de manera cada vez más certera el estar en la incertidumbre, indicaba una profesora. Vivimos en la individualidad, Ilevamos al aula ese manejo individual del contenido. Esto contrasta con un cirujano trabajando solo o un abogado, por ejemplo. Eso nos está llevando a una sociedad cada vez más deteriorada. Debemos ver cómo los equipos docentes deben jugar un papel importante, pues la Educación Superior es vital ya que crea ese profesional que está trabajando y definiendo su ciudadanía. Se aboga por el equipo, pero nos tienen dispersos, con un discurso que ha ido generando dualidades, concluía ella.

Una intervención resaltó entonces cómo el mundo de hoy es tan complejo y estamos tan acostumbrados a vivir aislados que cada quien es un emperador en su reino. Pero la solución de los problemas que son cada vez más difíciles está en la integración, en lo interdisciplinario, pues los problemas que tenemos son interdisciplinarios. Cada vez somos más seres humanos compartiendo los mismos bienes, de manera incluso agresiva.

El grupo reaccionó planteando que vivimos a espaldas de nuestra propia realidad y de esa manera la realidad nos arropa. La vida es cada vez más compleja y precisamente el discurso pedagógico es el que puede variar esa realidad a nivel global, pues es un discurso universal. En las universidades, para crear ese enfoque, tiene que haber una mística en esa dirección.

El grupo planteó la necesidad de que las universidades generen un espacio donde los profesores tengan la oportunidad de hablar de sus disciplinas y crear las posibilidades de que así las acciones se lleven a la práctica de manera conjunta.

Surgió la pregunta ¿es algo opcional trabajar en equipo? Es una realidad en la que las fronteras, los límites, las desconexiones las ponemos nosotros. El visualizarnos más importantes dentro de un grupo rompe la necesidad de articular, que es vital. Es prioritario, plantearon algunos profesores, una discusión sobre cuáles son las barreras que nos estamos poniendo, que impide que se dé el trabajo de equipo.

En relación a las experiencias del Cuaderno desde otras universidades, se resalta que una preocupación de las universidades desde hace unos años es el pasar de la individualidad a la cooperación. En las universidades existe una tendencia a romper con un proyecto aislado y llegar a un proyecto integrado, que las materias tengan interrelaciones con otras, para todos aportar al perfil del egresado de las carreras.

Para finalizar, se confirmó cómo desde el Centro de Desarrollo Profesoral, la PUCMM está haciendo un esfuerzo para un enfoque de trabajo en equipo e interdisciplinario entre sus docentes, desde la labor formativa en el Diplomado y en la Especialidad en Pedagogía Universitaria. Estos programas se enfocan en un proyecto pedagógico integrado con profesores de diversas disciplinas estudiando e investigando juntos sobre pedagogía y sobre su propia práctica.

Asistentes a la Peña: Julio Miguel Castaños, Daniel Pierre, Federico Fernández y Félix María Reyes, Derecho; Bárbara Campos, Matemáticas; Elisa Núñez, Lingüística Aplicada; Sara Güilamo, Psicología; Mariana Contreras, Comunicación Social; Leyda Brea y Alejandro Ascuasiati, Arquitectura; Amparo Fernández de Mejía, Educación; María Cantisano, Oliva Hernando y Luis Peña, Centro de Desarrollo Profesoral. 